\title{
J001 TRANSCRIPTIONAL REGULATION AND FUNCTION OF YEAST METALLOTHIONEINS
}

\author{
Pengbo Zhou, Keith A. Koch and Dennis J. Thiele
}

Department of Biological Chemistry, The University of Michigan Medical School, Ann Arbor, Michigan USA, 48109-0606

Metallothioneins (MTs) are low molecular weight, cysteine-rich metal binding proteins whose biosynthesis is activated, at the level of transcription, by a wide variety of metal ions and through specific metalresponsive promoter elements [1]. In the baker's yeast, Saccharomyces cerevisiae, a single MT gene exists, denoted $C U P 1$, which is transcriptionally activated only by the metals copper and silver, through the action of the metalloregulatory transcription factor ACE1. The ACE1 protein is a copper-activated sequence-specific DNA binding transcription factor which is constitutively synthesized and located in the $S$. cerevisiae nucleus poised for immediate activation of $C U P I$ gene transcription in response to toxic copper levels. In mammals and other higher eukaryotic species, MT protein isoforms are encoded by large gene families which are transcriptionally activated by many distinct metals via promoter metal regulatory elements (MREs) and, as yet unidentified, metal-responsive transcription factors [2]. To begin to understand the mechanisms by whiciı organisms with MT gene ramilies sense environmental metals and respond by activating transcription of MT gene families, we have begun to study metal-responsive transcription in the yeast Candida glabrata. $C$. glabrata harbors a single $M T-I$ gene, multiple tandemly amplified $M T$-II genes and a single, unlinked $M T-I I b$ gene. Using a surrogate genetic approach, we have cloned the gene from $C$. glabrata which encodes a copper metalloregulatory transcription factor, denoted AMT1, and have demonstrated that AMT1 binds to multiple sites in the promoter of each MT family member through which it activates MT gene transcription in response to copper. Interestingly, unlike $A C E I$ in $S$. cerevisiae, the $A M T I$ gene is subject to rapid positive transcriptional autoregulation in response to copper. This metalloregulatory transcription factor autoregulation plays a critical role in the copper homeostatic machinery, and suggests that organisms harboring MT gene families utilize similar, though distinct mechanisms for metal-responsive transcription.

1. J. H. R. Kagi and A. Schaffer, Biochemistry, 27, 8509 (1988).

2. D. J. Thiele, Nucleic Acids Research, 20, 1183 (1992). 